\section{(6) OPEN ACCESS}

\title{
Evolved design makes ThoraQuik safe and user friendly in the management of pneumothorax and pleural effusion
}

\author{
Sridhar Rathinam, ${ }^{1,2}$ Sophia Grobler, ${ }^{1}$ Antony Bleetman, ${ }^{3}$ Thomas Kink, ${ }^{4}$ \\ Richard Steyn ${ }^{1}$
}

${ }^{1}$ Regional Department of Thoracic Surgery, Birmingham Heartlands Hospital, Birmingham, UK

${ }^{2}$ Department of Thoracic Surgery, University Hospitals of Leicester, Leicester, UK ${ }^{3}$ Department of Emergency Medicine, Birmingham Heartlands Hospital, Bordesley Green East, Birmingham, UK ${ }^{4}$ Medical Device Innovations (MDi) Limited, Daresbury Innovation Centre, Daresbury Science and Innovation Campus, Halton, Cheshire

\section{Correspondence to} Dr Sridhar Rathinam, University Hospitals of Leicester, Groby Road, Leicester LE3 9QP, UK; srathinam@rcsed.ac.uk

Received 12 August 2012 Revised 28 November 2012 Accepted 4 December 2012 Published Online First 23 January 2013
To cite: Rathinam $S$ Grobler S, Bleetman A, et al. Emerg Med J 2014;31: 59-64.

\section{ABSTRACT}

Background We have previously described the utility of ThoraQuik, a device designed to be fit for purpose for aspirations of pneumothorax and pleural effusions. We evaluated the safety, efficacy and operator handling of the evolved prototype, ThoraQuik II, which has a lesser profile and a spring loaded Veres needle for added safety.

Methods A prospective, observational clinical trial with ethics and MHRA approval was conducted in a single centre. Patients with diagnosed pneumothorax (including tension pneumothorax) and pleural effusion were consented and recruited. The ease of device introduction, penetration and ease of use were evaluated. Clinical and radiological improvements were the clinical endpoints and operator feedback was analysed.

Results 20 procedures were performed on patients (mean age: 63.4 years (range: $30-90$ years) with $75 \%$ male subjects) recruited between September 2008 and August 2009. Nine patients had pneumothorax (tension pneumothorax $n=4$ ) and 11 had pleural effusions. 19 patients completed the study with symptomatic and radiological resolution. One patient was withdrawn due to poor pain threshold disproportionate to the procedure. No complications were encountered. $68 \%$ had complete clinical and radiological resolution and $32 \%$ had partial resolution (these patients needed a definitive drain and hence were not aspirated to completion). The operator feedback in the study rated the device as very good or excellent in $90 \%$ patients. Conclusions Our study found the use of ThoraQuik II to be safe and easy in draining pneumothorax and pleural effusions. The changes to ThoraQuik II made it more user friendly.

\section{BACKGROUND}

Pneumothorax and pleural effusions present in patients in a variety of ways, as acute emergencies in trauma in prehospital settings or as subacute presentations in hospitals. Tension pneumothorax is particularly a fatal complication which can result in cardiovascular collapse and death. ${ }^{1}{ }^{2}$ The management of pneumothoraces in the prehospital setting is managed by needle decompression, simple thoracostomy or tube thoracostomy for emergency decompression depending on the settings and experience of the personnel. ${ }^{3}$ Needle decompression may be lifesaving; however, its efficacy is still controversial due to the variety of the devices used for decompression. ${ }^{4-7}$
In the hospital settings patients present with spontaneous pneumothorax and pleural effusion. The British Thoracic Society guidelines recommend needle aspiration of pneumothoraces in the first instance. $^{8}$ Thoracentesis is also performed in pleural effusion to establish a diagnosis. ${ }^{9}$

A variety of techniques and devices are available for diagnostic or therapeutic evacuation of abnormal pleural contents depending on their composition, presentation, complexity and the success of preceding techniques. ${ }^{10}$ Aspiration is commonly performed using either large bore needles or intravenous cannula. These are not designed for this purpose and present difficulties in maintaining sufficient patency over even short periods of time. ${ }^{11} 12$

We have reported our evaluation of ThoraQuik (Medical Device Innovations (MDi), Daresbury Innovation Centre, Halton, Cheshire, UK) which is designed for both hospital and prehospital drainage of pneumothorax and pleural effusions (figure 1). ${ }^{13}$ We recommended changes to the device to make it safer and user friendly. This led to the development of ThoraQuik II (MDi), which has the similar features as ThoraQuik I with an ergonomic profile and atraumatic Veres needle. We evaluated the safety, efficacy, operator handling and acceptability of the ThoraQuik II device (henceforth called ThoraQuik II in this manuscript) for the treatment of pneumothorax and pleural effusion.

\section{METHODS}

\section{Design}

A prospective, observational clinical trial with the Central Office of Research Ethics Committee and $\mathrm{R} \& \mathrm{D}$ approval was conducted to evaluate the ThoraQuik II device. The study was carried out both in acute and semiacute settings in the emergency department and thoracic surgery department on patients with pneumothorax (including tension pneumothorax) and pleural effusions.

The device prototypes were provided by the developers MDi. The Medicines and Healthcare Products Regulatory Agency approval was obtained as the ThoraQuik II is a prototype investigational device. The study was designed, conducted, analysed and reported by the clinicians of Birmingham Heartlands Hospital who do not have any financial interests in the product. All patients provided written informed consent to participate in the study. The operator feedback on clarity of instructions, ease of handling of the device and procedure satisfaction was evaluated in a prestructured pro forma . 


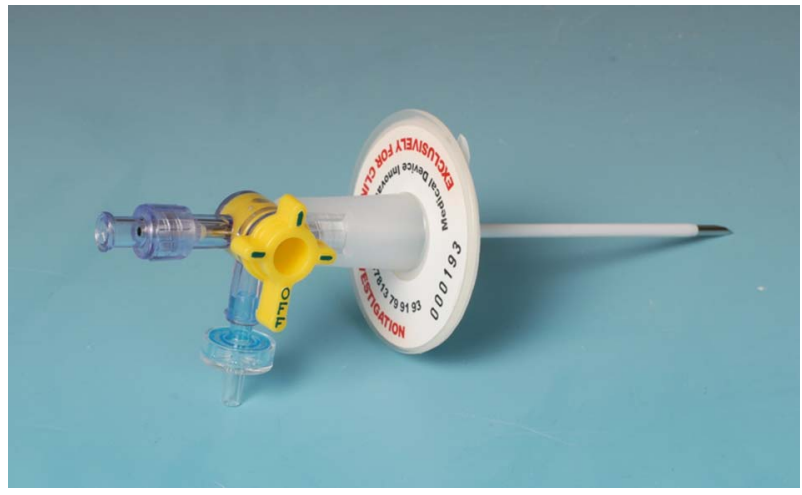

Figure 1 ThoraQuik I device.

\section{Device}

The ThoraQuik II is a sterile, single patient use device designed for aspiration consisting of a $9 \mathrm{~F}$ needle and an $8.5 \mathrm{G}$ cannula attached to a three-way lever tap and an adhesive securing flange (figure 2). It has an atraumatic Veres needle for added safety which is $10 \mathrm{~cm}$ long and a cannula measuring $9 \mathrm{~cm}$ from the flange. The body of the device has a central lumen with the distal end connected to the cannula and the proximal end of is attached to the three-way lever tap. This tap permits communication between the central lumen and a one-way duckbill valve permitting air and fluid trapped in the chest cavity to escape but not re-enter. The apertures may be reversibly opened by the lever tap, also enabling the connection and use of a syringe or tubing, via a Luer connection at the proximal end of the device. The device is supported in use on the patient's chest by the flange, and secured by a contact skin adhesive coating on the lower surface. Additionally, the device may be affixed to the patient's skin through sutures or safety pins, using the provided holes in the device flange. The device prototype was developed, tested and assessed by MDi. The clinical evaluation on patients was authorised by the Medicines and Healthcare Products Regulatory Agency.

\section{Primary objective}

The primary objective of the study was to evaluate the efficacy and safety of the ThoraQuik II device in the treatment of pneumothorax and pleural effusion.

\section{Secondary objective}

The secondary objective of the study was to obtain qualitative evaluations of operator handling of the ThoraQuik II device.

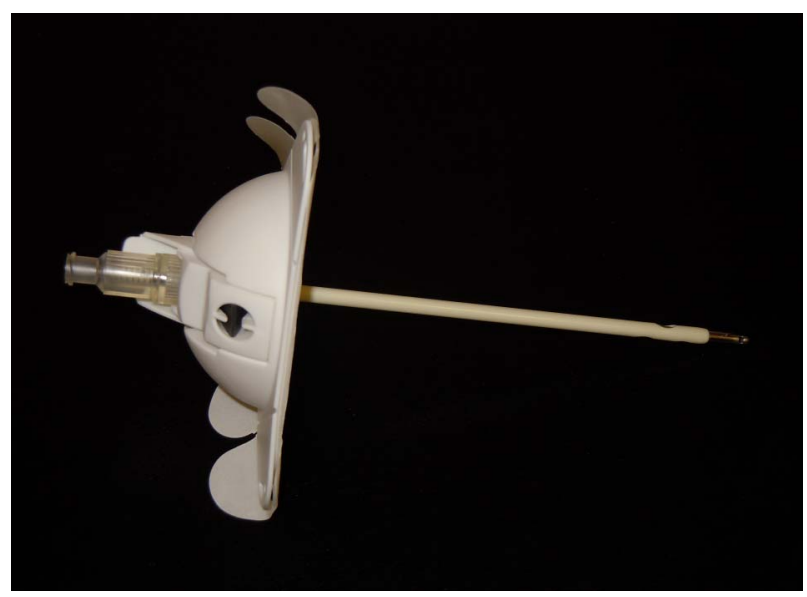

Figure 2 ThoraQuik II device.

\section{Procedure}

Utility and efficacy of the device

The indications for the device use were pleural effusion and pneumothorax (including tension pneumothorax). In the acute settings in the Emergency Department their prime mode of management was the ThoraQuik II for relief of symptoms, followed by definitive management with an intercostal drain where needed. In the thoracic surgery setting, patients with pneumothorax and pleural effusions as well as some patients waiting to have definitive surgery or chest drain insertion together were recruited. In the latter group, the ThoraQuik II was used to relieve the pleural effusion partially before a definitive procedure took place. All patients were counselled about the study and recruited after they had provided written informed consent on the form approved by the Ethics committee.

All the procedures were performed under local anaesthesia. The insertion of ThoraQuik II was done in a sterile and safe manner as described in our earlier publication. ${ }^{13}$ The choice of the site was in the triangle of safety for most patients and second anterior intercostal space in tension pneumothorax. Entry to the pleural space was confirmed by the aspiration of air/fluid into the syringe, and the needle was then gently withdrawn as the catheter was advanced. The tap on the device was turned to the one-way valve mode. A $50 \mathrm{ml}$ luer lock syringe replaced the $20 \mathrm{ml}$ syringe to facilitate more rapid aspiration with the tap switched back to the 'needle' position where necessary in pleural effusions. Aspiration of the contents was continued until the investigator considered it was appropriate to stop and/or the patient experienced a significant benefit. In cases where there was a requirement for a definitive drain, the ThoraQuik II device was removed and a chest drain was inserted as per standard procedure in the same site in the same sitting by extending the incision.

\section{Operator feedback}

Assessment of operator feedback regarding the clarity of instructions, ease of handling and procedure satisfaction was performed by a set questionnaire with a scoring system rating each section as excellent, good, adequate, poor and very poor.

Preuse assessment of the ThoraQuik II was split into two parts, Instructions and Packaging. The inuse assessment of the device examined the aspect of device functionality, such as the ease with which the syringe could be attached to the device, the syringe priming, the ease of penetration of the patient's chest by the device's needle and catheter, and the two-handed use. Finally, the overall device utility and ease were rated.

There was also a comment section to cover aspects of the device not covered in the questionnaire.

\section{RESULTS}

A total of 21 patients were screened and enrolled in the study between September 2008 and August 2009. One female patient withdrew consent before the procedure started and hence no data were collected. In all, 20 patients (15 male subjects) with an age range of 30-90 years (mean age of 66.6 years) were enrolled in the study. The height and weight distribution are illustrated in figure 3 . The majority of patients $(16,80 \%)$ were Caucasian $(80 \%)$; the remaining patients were Indian/Asian (3, $15 \%)$ and Oriental (1, 5\%). One patient has been treated twice with the ThoraQuik II device on each side on two independent occasions. One patient was withdrawn as unsuitable by the clinician, who following an injection of local anaesthetic at the site of device insertion experienced excessive pain disproportionate 
Figure 3 The height and weight distribution among the patients in the cohort.
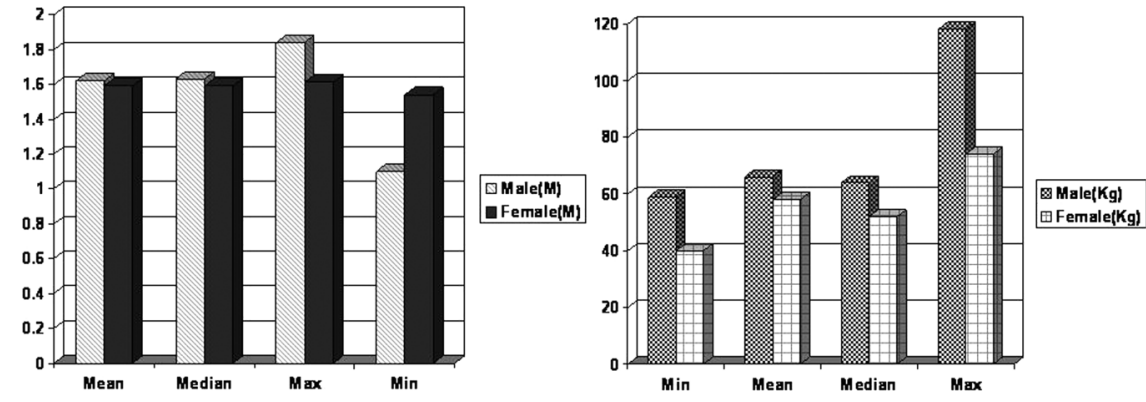

to the touching of the pleura with the needle. The patient was subsequently deferred for a formal chest drain, which also had been abandoned due to disproportionate pain. Of the 19 patients completed the study, 11 had pleural effusions and nine patients had pneumothorax (tension pneumothorax $n=4$ ) of which four were traumatic in origin (tension pneumothorax $n=2$ ), two were postsurgery (tension $n=1$ ), two were secondary pneumothoraces and one was postradio frequency ablation (tension $\mathrm{n}=1$ ).

\section{Device evaluation}

The device was evaluated by five investigators of varying seniority (two generic surgical trainees, one senior cardiothoracic resident, one consultant thoracic surgeon and one emergency medicine consultant). The senior resident (SR) and two consultants (AB and RSS) had evaluated the ThoraQuik I. The device placement by the surgical trainees was supervised by the senior resident.

\section{Preuse assessment}

Preuse assessment of the ThoraQuik II was split into two parts, Instructions and Packaging. The Instructions for Use, clarity of language and clarity of artwork ware rated as excellent in $100 \%, 100 \%$ and $95 \%$ of cases, respectively. Only in one case was the clarity of artwork rated good.

The robustness of the device packaging, the ease with which the devices could be stored, the package design and the clarity of storage instructions were considered to be excellent in 15

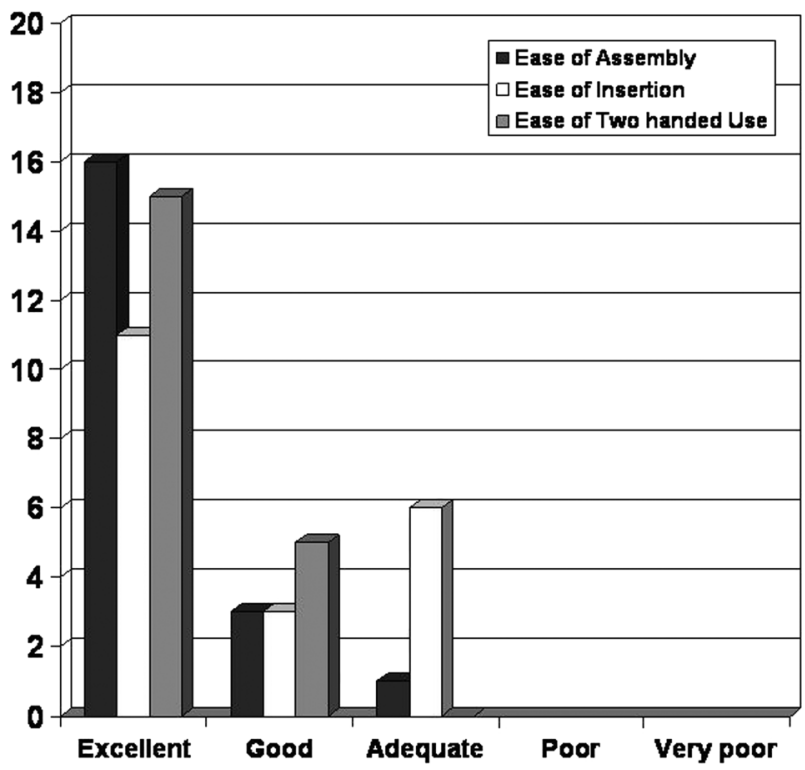

Figure 4 Clinician feedback regarding the device.
(71\%) of the cases. In the other cases, the assessment was good or adequate. The clarity of the expiry date and the ease of unpacking were rated excellent in 16 cases; again, they were rated good or adequate in the remaining cases.

\section{Inuse assessment}

The ease of device assembly was rated excellent in the majority of cases $(76 \%)$, good $(19 \%)$ or adequate $(5 \%)$ for all other cases. In one patient there was difficulty in removing the Veres needle subassembly after device insertion. Ease of syringe priming was rated excellent in $67 \%$ of the cases, good in $19 \%$ of all cases, adequate in $9 \%$ and poor in one case $(5 \%)$.

In the majority of cases the ease of insertion through the chest wall was rated excellent (52\%), while in the remaining cases, the rating was good $(19 \%)$ or adequate $(29 \%)$. In two patients there was wrinkling of the catheter on the skin during insertion.

The ease of two-handed use was rated excellent (71\%). In all other cases, it received the rating good (29\%). The site of insertion was rated excellent $(71 \%)$ or good $(29 \%)$ in all cases (figure 4).

\section{Device features}

Needle

In two cases of all 20 procedures (10\%), the needle got blocked during the treatment. However, this blockage could be easily removed as per instructions in the Instructions for Use.

The Veres needle performed as expected in all but one case where there was difficulty in removing the Veres needle subassembly after device insertion, but no reduced functionality for treatment was noted.

\section{Cannula}

In $18(90 \%)$ out of 20 cases, the cannula was deemed to perform appropriately. The bore diameter was rated wide enough in all cases. In four out of 20 cases (20\%), the cannula crimped during insertion at skin. However, there was no interference with the functionality of the device.

\section{One-way valve}

The one-way valve performed as expected in all cases and did not get blocked in any of the cases.

\section{Three-way tap}

The three-way tap operated as expected and intuitively in all cases. The marking on the tap was perceived and clear in indicating the current position in all cases. The tap became blocked in one instance $(5 \%)$, but again, could easily be cleared as per instructions. 
Figure 5 Outcomes with ThoraQuik II. (A) Pneumothorax before ThoraQuik II insertion, (B) postdrainage film showing resolution of pneumothorax (C) pleural effusion before drainage with ThoraQuik II and (D) postdrainage film showing resolution of pleural effusion.

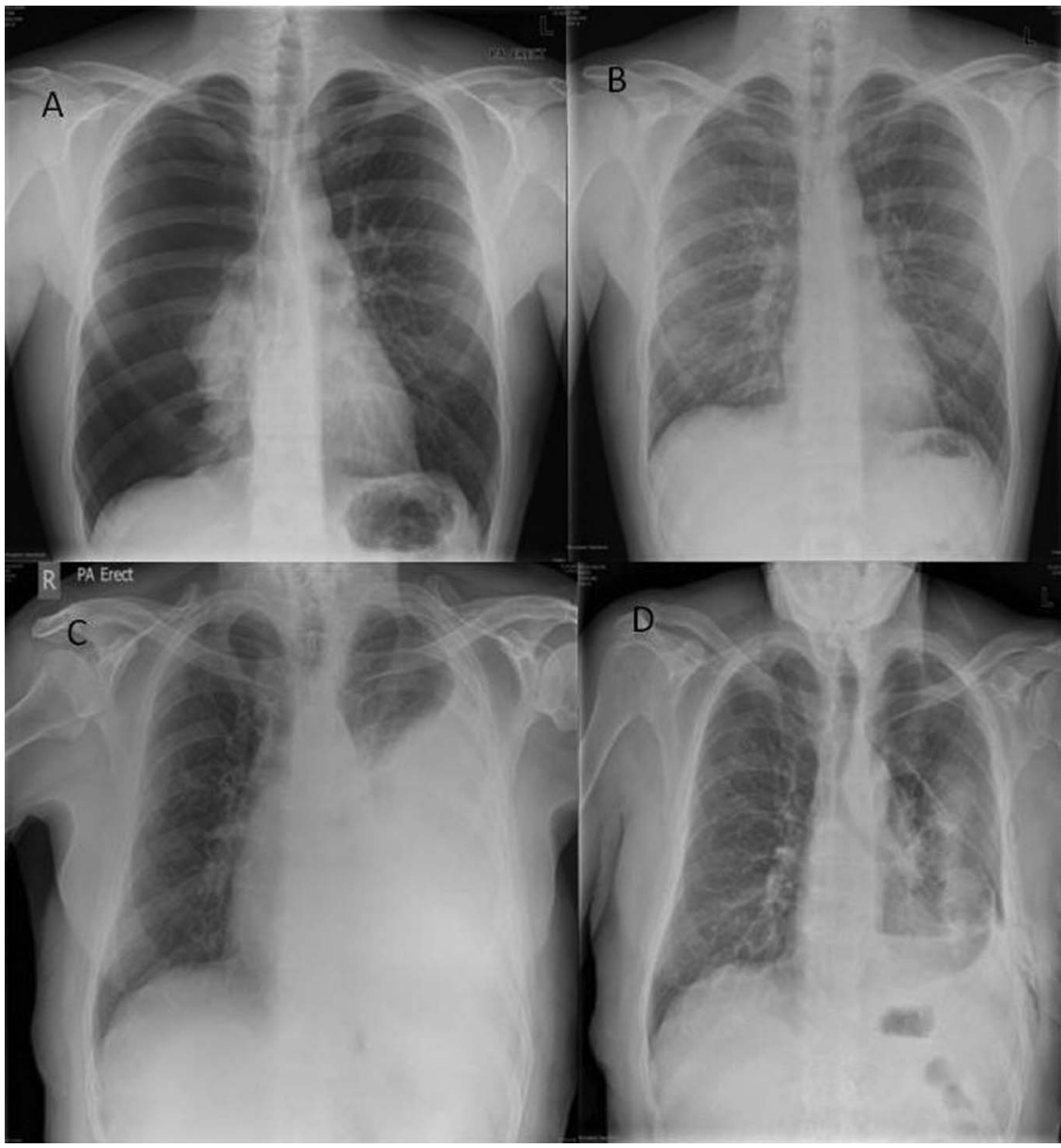

\section{Adhesive pad}

The release paper was easy to remove on all cases, the pad stuck properly to the skin as expected and the flexible wings helped securing the device in all cases. Only in one case was the device moving after fixation to the skin and in one case it had been secured additionally by a single stitch. This was done as a precaution and not required by device movement after fixation.

\section{Device breakages and/or damages during use}

In two cases (10\%), the syringe attachment/Luer lock broke during the treatment. However, no negative effect to the device functionality or treatment outcome occurred on those occasions. In one case, the removal of the Veres needle subassembly was difficult and the catheter got kinked during this process. Hence, this device had been discarded and a new device was used.

\section{Removal of the device}

The removal of the device at the end of the procedure was rated excellent in most cases (95\%) and good in one case.

\section{Duration of procedure}

The mean duration of the procedure, defined as the time from the preparation of insertion site to completion of decompression, was $13.8 \mathrm{~min}$ (range $5-40 \mathrm{~min}$ ); it was shorter in male (12.7 $\mathrm{min})$ compared with female patients (19.8 $\mathrm{min})$.

\section{Efficacy of device}

Overall, the device has been used to treat tension pneumothorax (n: 4), pneumothorax (n: 5) and pleural effusion (n: 12). There was complete radiological resolution in $60 \%$ of the patients and partial resolution in 40\% (figure 5). There was complete resolution of symptoms in $55 \%$ and partial resolution in $45 \%$ patients (table 1). It should be pointed out that partial resolution was in patients where a chest drain was planned as the definite procedure. In those cases, needle decompression was performed partially to enable safe insertion of the intercostal chest drain.

\section{Overall assessment}

The overall user assessment of the ThoraQuik II device was either excellent $(60 \%)$ or good $(40 \%)$ (table 2$)$. In cases of pleural effusion, fluid in excess of 2 litres has been removed successfully with ThoraQuik II.

\section{DISCUSSION}

Pneumothoraces and pleural effusion can cause significant symptoms and respiratory compromise. Pneumothorax can be

Table 1 Device efficacy

\begin{tabular}{|c|c|c|c|c|}
\hline & Male (N: 15) & Female (N: 5 ) & All & Outcome as intended \\
\hline \multicolumn{5}{|c|}{ Radiological resolution of pathology } \\
\hline Complete & $9(60 \%)$ & $3(60 \%)$ & $12(60 \%)$ & $100 \%$ \\
\hline Partial & $6(40 \%)$ & $2(40 \%)$ & $8(40 \%)$ & $100 \%$ \\
\hline \multicolumn{5}{|c|}{ Clinical resolution of pathology } \\
\hline Complete & $9(60 \%)$ & $2(40 \%)$ & $11(55 \%)$ & $100 \%$ \\
\hline Partial & $6(40 \%)$ & $3(60 \%)$ & $9(45 \%)$ & $100 \%$ \\
\hline
\end{tabular}


Table 2 Clinician feedback regarding the utility and efficacy of the device

\begin{tabular}{llllll}
\hline & Excellent & Good & Adequate & Poor & Very poor \\
\hline Ease of device assembly & 16 & 3 & 1 & & \\
Ease of penetration & 11 & 3 & 6 & \\
Two-handed use & 15 & 5 & & \\
Device performance & 12 & 8 & & \\
\hline
\end{tabular}

primary, secondary or traumatic and each condition is different in its presentation, complexity of management and definitive treatment. However, the early and immediate management of all these conditions has a common management modality which is needle aspiration and decompression. ${ }^{1} 4578$

Needle decompression of traumatic pneumothoraces and tension pneumothoraces prior to transfer to medical facilities is a practice which has been used for a long time. ${ }^{14}$ However, this is one of the controversial practices with concerns about its ability to decompress pneumothoraces as well as the efficacy questioned repeatedly. ${ }^{15}$

Needle aspiration is acknowledged as a relatively rapid intervention in the treatment of tension pneumothorax in the prehospital setting. ${ }^{1}{ }^{3-5}$ It has been shown that this procedure could be taught to non-medical professionals to save lives in tension pneumothoraces; ${ }^{16}$ however, there have been studies with radiological evidence reporting that needle thoracostomy only works in $50 \%$ of the instances due to the deficiency in catheter length. ${ }^{15} 1718$ Catheters which are longer than $4.5 \mathrm{cms}$ had a better outcome in needle decompression. ${ }^{18}$

The areas of concern are the success of the needles to traverse the chest wall and decompress the pneumothorax, and the lack of a preassembled custom made kit which is fit for purpose particularly in prehospital setting and emergency departments particularly when dealing with tension pneumothoraces can be fatal. ${ }^{11} 15$ The other concern with cannula decompression is the potential the risk of kinking and bending after insertion which may be life threatening in tension pneumothoraces. ${ }^{12}$ Although there are no published evidence showing the sensitivity of needle decompression, it is regularly taught as a 'rule out' investigation in the diagnosis of suspected tension pneumothorax. ${ }^{15}$

Some authors conclude that needle thoracocentesis is an unreliable means of decompressing the chest of an unstable patient and should only be used as a technique of last resort and recommend blunt dissection and digital decompression and advice insertion of a chest tube. ${ }^{5}$

Though the most common technique is needle aspiration and needle decompression, there are various devices which are used to aspirate and decompress pneumothoraces and pleural effusions starting from venous cannula to spinal needles to some custom made devices. ${ }^{19}$

A Cochrane review showed no significant difference between simple aspiration and intercostal tube drainage in the management of spontaneous pneumothoraces using immediate success rate, early failure rate, duration of hospitalisation, 1-year success rate and number of patients requiring pleurodesis at 1 year as endpoints. Moreover, the simple aspiration was associated with a reduction in the per cent of patients hospitalised when compared with intercostal tube drainage according to this review. ${ }^{10}$

Pleural effusions are collections of fluid in the chest which are again aspirated for diagnosis or decompression prior to definitive management by the way of a chest drain. ${ }^{9}$ Iatrogenic pneumothorax is a common complication of thoracentesis and frequently requires chest tube insertion. ${ }^{9}$ This may also be due to the fact that most often aspiration is performed with sharp needles or cannulas. Real-time ultrasonography use is a modifiable factor that reduces the pneumothorax rate. ${ }^{9} 20$

ThoraQuik I was designed on the concept of having a device with the length, the diameter and component constellation that will drain the pneumothorax and effusion rapidly. The added benefit of the device is the one-way disc valve which enables drainage without letting air into the pleural space. ${ }^{13}$ One negative aspect of the ThoraQuik I was the large calibre, unprotected sharp needle. The device also had a higher profile which, while not affecting the efficacy of the device, raised concerns that this may have a negative effect during patient transfer in real life emergencies. These design issues were altered and the next prototype device, the ThoraQuik II, has a Veres safety needle to avoid damage to underlying structures following penetration of the chest wall during insertion. It has a lower profile and a larger footprint providing a greater adhesive contact area and an even more flexible catheter. The catheter crimping and breakage were attributed by the manufacturers to the material strength as this was a small cohort of prototype and assured that it would be more robust in the final product.

Our evaluation has shown that ThoraQuik II is easy to insert to decompress pneumothoraces and aspirate pleural effusions and was better than its earlier prototype. The investigators felt more comfortable inserting the device. The new profile of the mushroom device had a significantly lower height and the larger adhesive area stabilised the device during transfer. ThoraQuik II has a role both in the emergency settings to decompress tension pneumothorax as well as in the inpatient setting for aspiration of pneumothoraces and thoracocentesis of effusions.

\section{CONCLUSIONS}

Our study found the use of ThoraQuik II to be safe and easy in draining pneumothorax (including tension pneumothorax) and pleural effusions. The changes to the first prototype device have made the device more user friendly and safer. We need to evaluate the efficacy of the device in larger numbers.

Acknowledgements MDi for providing the ThoraQuik device free of cost; Dr Pete Wall the Study Manager at MDi who was involved in the MHRA and ethics approval stage of the study; and the Consultant Thoracic Surgeons of Birmingham Heartlands Hospital who supported the study.

Contributors SR was involved with study design, collected the data, performed the data analysis and authored the manuscript. SG and AB collected data and coauthored the manuscript. TK managed the study for MHRA and coauthored the device specifics in the manuscript. RSS is the principal investigator, devised the study and coauthored the manuscript. All authors have read and approved the manuscript.

Competing interests The study was designed, conducted, analysed and reported by the clinicians of Birmingham Heartlands Hospital who do not have any financial interests in the product. The devices were provided free of cost by Medical Device Innovations (MDi).

Patient consent Obtained.

Ethics approval North Staffordshire Ethics Committee.

Provenance and peer review Not commissioned; externally peer reviewed.

Data sharing statement None. The complete report has been submitted to MHRA

Open Access This is an Open Access article distributed in accordance with the Creative Commons Attribution Non Commercial (CC BY-NC 4.0) license, which permits others to distribute, remix, adapt, build upon this work non-commercially, and license their derivative works on different terms, provided the original work is properly cited and the use is non-commercial. See: http://creativecommons.org/ licenses/by-nc/4.0/

\section{REFERENCES}

1 Leigh-Smith S, Davies G. Indications for thoracic needle decompression. J Trauma 2007:63:1403-4. 
2 McPherson JJ, Feigin DS, Bellamy RF. Prevalence of tension pneumothorax in fatally wounded combat casualties. J Trauma 2006;60:573-8.

3 Barton ED, Epperson M, Hoyt DB, et al. Prehospital needle aspiration and tube thoracostomy in trauma victims: a six-year experience with aeromedical crews. J Emerg Med 1995;13:155-63 .

4 Eckstein M, Suyehara D. Needle thoracostomy in the prehospital setting. Prehosp Emerg Care 1998;2:132-5.

5 Fitzgerald M, Mackenzie CF, Marasco S, et al. Pleural decompression and drainage during trauma reception and resuscitation. Injury 2008;39:9-20.

6 Waydhas C, Sauerland S. Pre-hospital pleural decompression and chest tube placement after blunt trauma: a systematic review. Resuscitation 2007:72:11-25.

7 Faruqi S, Gupta D, Aggarwal AN, et al. Role of simple needle aspiration in the management of pneumothorax. Indian J Chest Dis Allied Sci 2004:46:183-90.

8 MacDuff A, Arnold A, Harvey J. Management of spontaneous pneumothorax: British Thoracic Society Pleural Disease Guideline 2010. Thorax 2010;65(Suppl 2):ii18-31.

9 Gordon CE, Feller-Kopman D, Balk EM, et al. Pneumothorax following thoracentesis: a systematic review and meta-analysis. Arch Intern Med 2010;170:332-9.

10 Wakai A, O'Sullivan RG, McCabe G. Simple aspiration versus intercostal tube drainage for primary spontaneous pneumothorax in adults. Cochrane Database Syst Rev 2007;24:CD004479. doi:10.1002/14651858.CD004479.
11 Britten S, Palmer SH, Snow TM. Needle thoracocentesis in tension pneumothorax: insufficient cannula length and potential failure. Injury 1996;27:321-2.

12 Zengerink I, Brink PR, Laupland KB, et al. Needle thoracostomy in the treatment of a tension pneumothorax in trauma patients: what size needle? J Trauma 2008:64:111-14

13 Rathinam S, Quinn DW, Bleetman A, et al. Evaluation of ThoraQuik: a new device for the treatment of pneumothorax and pleural effusion. Emerg Med Jo 2011;28:750-3.

14 Lee C, Revell M, Porter K, et al. The prehospital management of chest injuries: a consensus statement. Faculty of pre-hospital care, Royal College of Surgeons of Edinburgh. Emerg Med J 2007;24:220-4.

15 Leigh-Smith S, Harris T. Tension pneumothorax-time for a re-think? Emerg Med J 2005;22:8-16.

16 Sztajnkrycer MD. Needle thoracostomy by non-medical law enforcement personnel: preliminary data on knowledge retention. Prehosp Disaster Med 23:553-7.

17 Stevens RL, et al. Needle thoracostomy for tension pneumothorax: failure predicted by chest computed tomography. Prehosp Emerg Care 13:14-17.

18 Ball CG, et al. Thoracic needle decompression for tension pneumothorax: clinical correlation with catheter length. Can Jo Surg 2010;53:184-8.

19 Wayne MA, McSwain NE. Clinical evaluation of a new device for the treatment of tension pneumothorax. Ann Surg 1980;191:760-2.

20 Daniels CE, Ryu JH. Improving the safety of thoracentesis. Curr Opin Pulm Med 2011;17:232-6 